\title{
PRIME IDEALS IN UNIFORM ALGEBRAS
}

\author{
WILLIAM E. DIETRICH, JR.
}

ABstract. A uniform algebra on a compact metric space has infinite Krull dimension and exactly $2^{c}$ nonmaximal prime ideals.

A subalgebra of the continuous, complex-valued functions $C(X)$ on a compact Hausdorff space $X$ which separates the points of $X$, contains the constant functions and is closed in the uniform norm \|\|$_{\infty}$ is called a uniform algebra. This topic has been elaborated for more than two decades (e.g. [4]), but with little attention to algebraic questions. In the classical disc algebra, for example, 0 is a prime ideal and no nonzero prime ideal can lie properly in any maximal ideal determined by an interior point of the disc: does the disc algebra even have a nonzero, nonmaximal prime? An interpolation method used here implies that each boundary maximal ideal will contain $2^{c}$ such ideals, arranged in $2^{c}$ nonoverlapping infinite chains [Theorem 1]. Even more, every uniform algebra on a first countable space has at least one maximal ideal with this property [Theorem 2]; in particular, its Krull dimension is infinite.

For a subset $B$ of a uniform algebra $A$, let $Z(B)$ stand for the set of common zeros of the functions in $B$ and for $p \in X$, let $I_{p}$ denote the maximal ideal of functions in $A$ which vanish at $p$. Denote the Choquet boundary of $A$ by $\partial A[1$, p. 81].

THEOREM 1. Suppose $A$ is a uniform algebra and $J$ is an ideal of $A$. If $p$ is a peak point for $A$ which is not isolated in $Z(J) \cap \partial A$, there are $2^{c}$ pairwise disjoint, infinite ascending chains of prime ideals of $A$ with each prime containing $J$ and densely contained in $I_{p}$. In particular, $\operatorname{krull} \operatorname{dim} A / J=\infty$.

Proof. Choose $f \in A$ with $f(p)=1$ and $|f(x)|<1$ if $x \neq p$. Inductively select $p_{n} \in Z(J) \cap \partial A$ so that $\left|1-f\left(p_{n}\right)\right|<\min \left\{1 / n,\left|1-f\left(p_{n-1}\right)\right|\right\} . p$ is the only possible accumulation point $q$ of the set $\left\{p_{n}\right\}$. For $f(q)$ is an accumulation point of the distinct points $\left\{f\left(p_{n}\right)\right\}$; since $f\left(p_{n}\right) \rightarrow 1, f(q)=1$ so actually $q=p$. Thus $K=\left\{p_{n}\right\} \cup\{p\}$ is compact, $f$ is a homeomorphism of $K$ onto $f(K)$ and $p_{n} \rightarrow p$.

Received by the editors December 26, 1972 and, in revised form, April 27, 1973.

AMS (MOS) subject classifications (1970). Primary 46J10, 46J20; Secondary 16A12. Key words and phrases. Prime ideal, uniform algebra, peak point, Choquet boundary, interpolating sequence.

(c) American Mathematical Society 1974 
$A \mid K$ is dense in $C(K)$. Take a Borel measure $\mu$ on $K$ which annihilates $A \mid K$; since $f^{n} \rightarrow \chi_{\{p\}}$ boundedly, $0=\int_{K} f^{n} d \mu \rightarrow \mu(\{p\})$. Also because $p_{n} \rightarrow p$, each $p_{n}$ has a neighborhood $V_{n}$ which misses $K-\left\{p_{n}\right\}$; since $p_{n}$ is a strong boundary point of $A[1,2.3 .4]$, there is some $k_{n} \in A$ with $\left\|k_{n}\right\|_{\infty}=1=$ $k_{n}\left(p_{n}\right)$ and $\left|k_{n}\right|<1$ off $V_{n}$. Given any $\varepsilon>0$ we can take a high enough power of $k_{n}$ to obtain some $g_{n} \in A$ with $g_{n}\left(p_{n}\right)=1$ and $\left|g_{n}\right|<\varepsilon$ on $K-\left\{p_{n}\right\}$.

$$
0=\left|\int_{K} g_{n} d \mu\right| \geqq\left|\mu\left(\left\{p_{n}\right\}\right)\right|-\varepsilon\|\mu\|,
$$

and letting $\varepsilon \rightarrow 0$ we see that $\mu\left(\left\{p_{n}\right\}\right)=0$. Thus

$$
|\mu|(K)=|\mu(\{p\})|+\sum_{n=1}^{\infty}\left|\mu\left(\left\{p_{n}\right\}\right)\right|=0 ;
$$

by the Hahn-Banach theorem, $A \mid K$ is dense in $C(K)$.

$K$ is a closed set which is a countable union of peak points in the weak sense, so that Glicksberg's peak set theorem [4, II. 12.7, p. 58] implies $K$ is an intersection of peak sets. Thus $A \mid K$ is closed in $C(K)$; in fact then, $A \mid K=C(K)$.

$K$ is homeomorphic to $N_{\infty}$, the one point compactification of the natural numbers, and composing the induced isomorphism $C(K) \cong C\left(N_{\infty}\right)$ with restriction $A \rightarrow C(K)$, we obtain an algebra homomorphism $\Phi$ of $A$ onto $C\left(N_{\infty}\right)$ such that $J \subset \operatorname{ker} \Phi$ and $\Phi\left(I_{p}\right)=M_{\infty}=\left\{f \in C\left(N_{\infty}\right): f(\infty)=0\right\}$. According to $\left[5,14 \mathrm{G}\right.$, p. 213] there are $2^{c}$ maximal chains of prime ideals of $C_{r}\left(N_{\infty}\right)$ [the real-valued continuous functions on $N_{\infty}$ ] contained in $M_{\infty}^{r}=M_{\infty} \cap C_{r}\left(N_{\infty}\right)$, and any two chains have only $M_{\infty}^{r}$ in common. For any such chain $\mathscr{C}$, set $\mathscr{C}^{*}=\left\{\Phi^{-1}(P+i P): P \in \mathscr{C}, P \neq M_{\infty}^{r}\right\}$. Since $P \rightarrow P+i P$ is a lattice preserving one-to-one correspondence between the primes of $C_{r}\left(N_{\infty}\right)$ and those of $C\left(N_{\infty}\right)[3,1.1], \mathscr{C}^{*}$ is a chain of prime ideals of $A$ contained in $\Phi^{-1}\left(M_{\infty}\right)=I_{p}$ and containing $J$; plainly if $\mathscr{D}^{*}$ is any other such chain, $\mathscr{D}^{*} \cap \mathscr{C}^{*}=\varnothing$. Each chain $\mathscr{C}^{*}$ is infinite ascending since $P \rightarrow \Phi^{-1}(P+i P)$ is a lattice preserving bijection and $\mathscr{C}$ is infinite ascending. For otherwise there is some largest $P \in \mathscr{C}$ properly contained in $M_{\infty}^{r}$ and because $\mathscr{C}$ is maximal, there is no prime ideal of $C_{r}\left(N_{\infty}\right)$ strictly between $P$ and $M_{\infty}^{r}:$ a violation of $[2,3.2$, p. 71$]$.

Finally each $Q \in \mathscr{C}^{*}$ is dense in $I_{p}$. Indeed the prime $P=\Phi(Q)$ is dense in $M_{p}[2,1.5,1.8]$ : given $f \in I_{p}$, there are $g_{n} \in P$ with $\left\|g_{n}-\Phi(f)\right\|_{\infty} \rightarrow 0$. Since $K$ is a peak interpolation set, there are $h_{n} \in A$ with $\left\|h_{n}\right\|_{X}=$ $\left\|g_{n}-\Phi(f)\right\|_{\infty}$ and $\Phi\left(h_{n}\right)=g_{n}-\Phi(f)$. Thus $h_{n}+f \in \Phi^{-1}(P)=Q$ and $\left\|h_{n}+f-f\right\|_{X} \rightarrow 0$.

Of course none of the nonmaximal primes constructed above is closed. This is to be expected since in the disc algebra, for example, Rudin's 
characterization of the closed ideals $[6, p .85]$ implies that 0 is the only nonmaximal closed prime.

In practice a uniform algebra may only have peak points isolated in its Šilov boundary, or because no point has a countable neighborhood base, even none at all. Nevertheless we have

THEOREM 2. A uniform algebra $A$ on an infinite first countable space $X$ has a maximal ideal which contains $2^{c}$ pairwise disjoint infinite chains of prime ideals.

Proof. Suppose $\partial A$ is a discrete subspace of the Silov boundary $\Gamma$ of $A$. Then each $p \in \partial A$ is a peak point $[1,2.3 .1]$ which is open in $\Gamma:$ if $f$ peaks at $p, f^{n} \rightarrow \chi_{\{p\}}$ uniformly on $\Gamma$ so $1-\chi_{\{p\}} \in A$ peaks (in $\Gamma$ ) on $\Gamma-\{p\}$. Since $\Gamma$ is infinite [otherwise $A \cong A \mid \Gamma$ is finite-dimensional and because $A$ separates point, $X$ is finite], $\mathscr{F}=\{\Gamma-F: F \subset \partial A$ finite $\}$ is a family of nonvoid closed subsets of $\Gamma$ with the finite intersection property, so $P=\bigcap \mathscr{F}$ is a generalized peak set for $A \mid \Gamma$. By a theorem of Bishop $[1,2.4 .6$, p. 105] $P$ contains a generalized peak point $p \in \partial A \mid \Gamma=\partial A$. Thus $p \in \Gamma-\{p\}$, a contradiction.

We conclude that $\partial A$ contains a point $p$ nonisolated in $\partial A . p$ is a peak point which is the limit of an infinite sequence on $\partial A$; the result follows from the proof of Theorem 1 with $J=0$.

Notice if $X$ is actually metric (and hence separable), $A$ will have cardinality $c$, so that Theorem 2 implies

COROllary 1. A uniform algebra on an infinite metric space has infinite Krull dimension and exactly $2^{c}$ nonmaximal prime ideals.

The following answers a question of M. Weiss [8, p. 94].

COROLlaRY 2. In a uniform algebra on an infinite first countable space, not every finitely generated ideal is principal.

Proof. Otherwise, the primes contained in a fixed maximal ideal form a chain [5, 14L, p. 214] in violation of Theorem 2.

EXAMPLE. $H^{\infty}$, the bounded analytic functions on the open unit disc $\Delta$, considered as a uniform algebra on its maximal ideal space $M$ has no peak points and no point of $M-\Delta$ has a countable neighborhood base: Theorems 1 and 2 do not apply. Nonetheless, suppose $q \in M-\Delta$ lies in the closure of a Carleson-Newman interpolating sequence $S \subset \Delta: H^{\infty} \mid S=l^{\infty}=$ $C^{*}(S)$. Then cl $S$ is homeomorphic to $\beta S$, the Stone-Cech conpactification of $S\left[6\right.$, p. 205], so that actually $H^{\infty} \mid \beta S=C(\beta S)$. Since $S$ is realcompact, there is some $f \in C_{r}(\beta S)$ with $f(q)=0$ and $|f|>0$ on $S$ [5, p. 119]. $f$ cannot vanish on any neighborhood of $q$ and assuming the continuum hypothesis, it follows that the maximal ideal $M_{a}$ of $C_{r}(\beta X)$ determined by $q$ contains a 
chain of at least $2^{c}$ prime ideals $[5,14.19$, p. 204]. Because restriction is a homomorphism of $H^{\infty}$ onto $C(\beta S)$ which takes $I_{q}$ onto $M_{a}+i M_{a}, I_{a}$ contains a chain of $2^{c}$ prime ideals of $H^{\infty}$; in particular, krull $\operatorname{dim} H^{\infty}=\infty$.

Although interpolating sequences exist in profusion [6, p. 204], not every $q \in M-\Delta$ lies in the closure of such a set; in fact Hoffman has shown this happens exactly when the Gleason part for $q$ is nontrivial [7, 5.5, p. 101]. Since $H^{\infty}$ is logmodular on the maximal ideal space $X$ of $L^{\infty}$, each point of $X$ is a one point part; and there are others [4, Example 3, p. 162]. The prime structure at these points is not known, but things are clear elsewhere: if $q \in \Delta$, a routine order of zero argument shows that 0 is the only nonmaximal prime of $H^{\infty}$ in $I_{a}=(z-q) H^{\infty}$; if $q \in M-\Delta$ has a nontrivial part, $I_{a}$ contains exactly $2^{c}$ nonmaximal primes. Indeed, Carleson's corona theorem makes $M$ separable, so that $H^{\infty}$ has cardinality $c$ : even $H^{\infty}$ has exactly $2^{c}$ nonmaximal primes.

For $|\lambda|=1$ the fiber $M_{\lambda}=\{\phi \in M: \phi(z)=\lambda\}$ is a peak set for $H^{\infty}$, so that $A_{\lambda}=H^{\infty} \mid M_{\lambda}$ is a uniform algebra. There is an embedding $\psi: \Delta \rightarrow M_{\lambda}$ so that $A_{\lambda} \mid \psi(\Delta) \cong H^{\infty}\left[6\right.$, p. 168], and the composite $A_{\lambda} \rightarrow A_{\lambda} \mid \psi(\Delta) \rightarrow H^{\infty} \rightarrow$ $C(\beta S)$ makes $C(\beta S)$ a homomorphic image of $A_{\lambda}$. Therefore $A_{\lambda}$ will also contain exactly $2^{c}$ nonmaximal prime ideals and will have infinite Krull dimension.

\section{REFERENCES}

1. A. Browder, Introduction to function algebras, Benjamin, New York, 1969. MR 39 \#7431.

2. W. Dietrich, On the ideal structure of $C(X)$, Trans. Amer. Math. Soc. 152 (1970), 61-77. MR 42 \#850.

3. —_, Ideals in convolution algebras on Abelian groups, Pacific J. Math. (to appear).

4. T. Gamelin, Uniform algebras, Prentice-Hall, Englewood Cliffs, N.J., 1969.

5. L. Gillman and M. Jerison, Rings of continuous functions, University Series in Higher Math., Van Nostrand, Princeton, N.J., 1960. MR 22 \#6994.

6. K. Hoffman, Banach spaces of analytic functions, Prentice-Hall Series in Modern Analysis, Prentice-Hall, Englewood Cliffs, N.J., 1962. MR 24 \#A2844.

7. - Bounded analytic functions and Gleason parts, Ann. of Math. (2) 86 (1967), 74-111. MR 35 \#5945.

8. M. Weiss, Some separation properties in sup-norm algebras of continuous functions, Function Algebras (Proc. Internat. Sympos. on Function Algebras, Tulane Univ., 1965), Scott-Foresman, Chicago, Ill., 1966, pp. 93-97. MR 33 \#1756.

Department of Mathematics, University of Texas, Austin, TeXas 78712 